\title{
Measurement of Averaged Heat Transfer Coefficients in High-Pressure Vessel during Charging with Hydrogen, Nitrogen or Argon Gas*
}

\author{
Peter Lloyd WOODFIELD**, Masanori MONDE** and Yuichi MITSUTAKE*** \\ **Institute of Ocean Energy, Saga University, \\ 1 Honjo-machi, Saga 840-8502, Japan \\ E-mail: monde@me.saga-u.ac.jp \\ *** Department of Mechanical Engineering, Saga University, \\ 1 Honjo-machi, Saga 840-8502, Japan
}

\begin{abstract}
Heat transfer to the wall of a small pressure vessel during filling with some different kinds of gas was investigated experimentally. The vessel was orientated vertically with the inlet at the top. The space-averaged Nusselt number for the curved wall was found to be a function of both the Reynolds and Rayleigh numbers indicating a mixed convection heat transfer situation. A correlation is proposed for the heat transfer coefficient during charging of the vessel. For the six positions where measurements were taken, the local heat transfer coefficient typically did not differ from the space-averaged value by more than about 30 percent. Measurements were also taken during discharging to atmospheric pressure. For discharging, some of the data was found to agree with a correlation for natural convection in cylindrical geometry. Local Nusselt numbers for discharging tended to be higher towards the bottom of the vessel.
\end{abstract}

Key words: Pressure Vessel, Heat Transfer Coefficient, Hydrogen, Nitrogen, Argon

\section{Introduction}

Compressed hydrogen gas is presently the most popular choice for a fuel storage mode for vehicles in use in hydrogen demonstration projects around the world. The challenge with this method is that either a large volume or high pressures are required to store the desired three to five kilograms of hydrogen on-board ${ }^{(1) \sim(3)}$. This is because the hydrogen molecule is so light even though the energy per unit mass is high. Using liquid hydrogen ${ }^{(1)}$ overcomes the overall energy density problem but a cryogenic cooling system is required. Metal hydrides are another promising alternative but further development is still needed to make them competitive with compressed hydrogen gas technology.

Commercially available pressure vessels are presently designed to contain compressed hydrogen gas at pressures of $35 \mathrm{MPa}$ to $70 \mathrm{MPa}$ which are considerably higher than the typical maximum of $20 \mathrm{MPa}$ for compressed natural gas vehicles. To achieve these high pressures without using an excessively heavy cylinder for the vehicle, use is made of carbon fiber reinforced plastic composite (CFRP) materials ${ }^{(3),(4)}$. Commercially manufactured CFRP material is very strong and light-weight but has the downside that it is limited to operation at temperatures typically below about $85^{\circ} \mathrm{C}$ to meet safety regulations. Unfortunately, particularly on a hot summer's day, it is possible to exceed this temperature during semi-adiabatic compression of hydrogen gas as it flows from the filling station to a 
vessel being charged from atmospheric pressure to $35 \mathrm{MPa}$.

For practical design of suitable vessels and design of the hydrogen filling station itself, it is necessary to be able to predict accurately the temperatures of the vessel wall and gas during charging. Recently, Monde et al. ${ }^{(5)}$ showed that a simple thermodynamic model that assumes perfectly stirred conditions for the gas could give a useful estimate of the temperature inside a stainless steel hydrogen vessel during charging to $35 \mathrm{MPa}$. In their model, heat was transferred to the wall via convection and a one-dimensional unsteady heat conduction equation for the wall was solved simultaneously with a heat balance for the gas side. A deficiency with the model is that it is necessary to assume a space-averaged heat transfer coefficient between the gas and inside wall which may be different depending on the filling rate, size and geometry of the vessel. There is little data presently available in the open literature relating to space-averaged heat transfer coefficients for this situation since many different flow configurations are possible for flow in an enclosure.

As will be shown below, heat transfer in the cylinder is best described as mixed convection. Forced convection results from the jet of gas entering the vessel and natural convection occurs simultaneously due to the difference in temperature between the gas and the wall. For pure natural convection in cylinders Daney ${ }^{(6)}$ developed a correlation for the Nusselt number as a function of the Rayleigh number suitable for turbulent flow. He demonstrated that the same correlation is applicable for horizontal and vertical cylinders and also for spherical and hemispherical containers. Evans and Stefany ${ }^{(7)}$ could correlate their data for natural convection in cylindrical enclosures using a simple relation similar to what is commonly used for laminar natural convection to vertical walls. A number of numerical studies have been performed for natural convection in cylindrical enclosures ${ }^{(8) \sim(13)}$. Lin and Armfield ${ }^{(8),(9)}$ calculated flow fields and heat transfer in both cylindrical and $2 \mathrm{D}$ rectangular enclosures after a step change in the temperature of the sidewalls. They found many similarities between the heat transfer flow patterns for the two geometries and their calculated data scaled well with $R a^{1 / 4}$, which is typical behavior for laminar flow. Lemembre and Petit ${ }^{(10)}$ and Kwak et al. ${ }^{(11)}$ also performed calculations for laminar flow. Holzbecher and Steiff ${ }^{(12)}$ and Papanicolaou and Belessiotis ${ }^{(13)}$ included turbulence models in their simulations of natural convection in the cylinder.

For mixed convection heat transfer in cylindrical geometry, the most widely studied case is the heated vertical tube. Jackson et al. ${ }^{(14),(15)}$ provide a review and some detailed discussion of mixed convection in vertical tubes. The effect of combining forced and free convection may be additive or subtractive depending on whether the buoyancy assists or opposes the motion. It is interesting that turbulent and laminar mixed convection flows can show opposite trends in this respect ${ }^{(15)}$.

In the present article we report results from direct measurement of Nusselt numbers for a pressure vessel during charging and discharging with some different kinds of gases. The abovementioned studies are used to guide development of a correlation for mixed convection heat transfer in the pressure vessel. It is worth noting that the pressure vessel and gas flow rates used in this study are smaller than what is typically used in hydrogen vehicle applications. However, the use of the heavier gases, nitrogen and argon, results in a range of Reynolds and Rayleigh numbers that is also appropriate for much larger hydrogen storage vessels.

\section{Nomenclature}
$A:$ surface area of copper strip in contact with gas
$c:$ specific heat of copper strip
$c_{\mathrm{p}}$ : constant pressure specific heat of gas
$d$ : inside diameter of inlet to pressure vessel
$D$ : inside diameter of pressure vessel
$g$ : acceleration due to gravity 


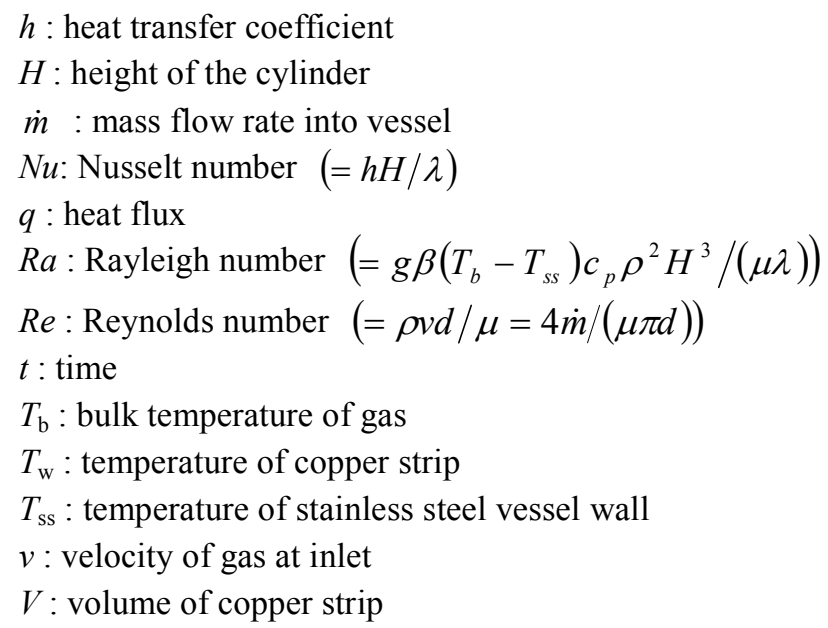

\section{Greek}

$\beta$ : volumetric thermal expansion coefficient

$\lambda$ : thermal conductivity

$\mu$ : absolute viscosity

$\rho:$ density

$\tau:$ dimensionless time defined by Eq. (2)

\section{Experiment}

\subsection{Test cylinder}

Figure 1 shows a diagram of the test cylinder. The vessel wall is constructed using stainless steel and is designed for pressures up to $40 \mathrm{MPa}$. The volume of the vessel is about $1.4 \mathrm{~L}$ with an inside diameter of $93.7 \mathrm{~mm}$ ( $75 \mathrm{~mm}$ when the PVC insert is included) and inside height of $212.7 \mathrm{~mm}$. The thickness of the stainless steel wall is about $30 \mathrm{~mm}$. Hydrogen enters the vessel through a $10 \mathrm{~mm}$ diameter hole in the center of one end of the vessel. The other end is sealed with a $92 \mathrm{~mm}$ thick detachable lid. During the experiment the vessel is orientated so that the hydrogen inlet was at the top.

In order to determine heat transfer coefficients it is necessary to measure the wall surface temperature, heat flux and the gas temperature. Since the thermal inertia of the vessel wall in Fig. 1 is large, previous measurements showed that the stainless steel wall temperature only changes a few Kelvin during charging ${ }^{(5)}$. We consider that this is insufficient to accurately determine the heat flux to the wall by calculation from direct measurement of the stainless steel surface temperature. For this reason we decided to mount a row of copper strips on a smaller diameter polyvinyl chloride (PVC) tube and insulate them from direct thermal contact with the stainless steel surface as shown in Fig. 1. By measuring the temperature of the copper strip as a function of time we have enough information to determine the surface temperature and heat flux to the inner surface of the strip. Simultaneous measurement of the bulk gas temperature enables us to estimate the heat transfer coefficient spatially averaged over the width of each copper strip. The rubber insulation and PVC tube also have the overall effect of retarding the heat transfer to the stainless steel vessel wall and therefore for the same final pressure the gas temperature rises more significantly during the present experiment than in our previous study ${ }^{(5)}$. We expect the heat transfer coefficients as a function of the Reynolds and Rayleigh numbers will be similar with and without the insert.

Figure 2 shows the geometry of a single copper strip used to provide heat flux and temperatures required to estimate the heat transfer coefficient. The thickness is $5 \mathrm{~mm}$, height 20 to $24 \mathrm{~mm}$ and circumferential length about $80 \mathrm{~mm}$. Even if we base the Biot number on the height instead of the thickness, the engineering limit of $B i<0.1$ is still valid 


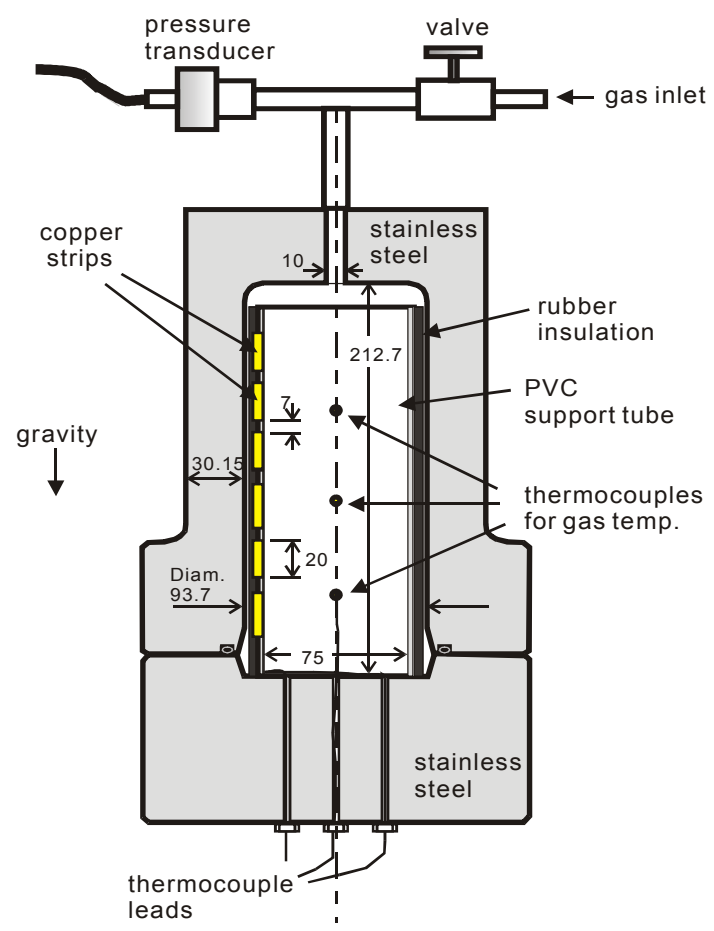

Fig. 1 Test cylinder (units: mm)

for heat transfer coefficients as high as $1500 \mathrm{~W} / \mathrm{m}^{2} \mathrm{~K}$. Therefore we consider it is safe to treat the strip as a lumped mass so that the temperature measured by the $1 \mathrm{~mm}$ sheath diameter, chromel/alumel thermocouple can be assumed to be equivalent to the surface temperature. Also the heat flux to each copper strip can be obtained by differentiation of the thermocouple reading with respect to time and then multiplying by the heat capacity of the copper mass. In the present analysis it was assumed that heat was transferred only to the inside surface of the copper strip exposed to the gas $(64 \mathrm{~mm} \times 20 \mathrm{~mm})$. The lumped mass used in the calculation included the lugs on the ends of the strips that were used to attach the copper to the supporting PVC tube. The heat flux reported in this article should be considered as an average over the surface of the copper strip. The inner surface of each strip is mounted flush with the PVC wall to provide minimal disturbance to the flow. Notice also in Fig. 1 that there is a gap of $7 \mathrm{~mm}$ between neighboring copper strips. This gap has a disadvantage that there may be some disturbance to the thermal boundary layer development. However, the low thermal conductivity of the PVC material ensures that strips are insulated from each other making it possible to obtain a heat flux measurement for each individual strip.

Gas temperatures were measured at three positions along the axis of the cylinder: 69 $\mathrm{mm}, 118 \mathrm{~mm}$ and $168 \mathrm{~mm}$ from the gas inlet. The average of these three was taken as representative of the bulk gas temperature at any point in time. The thermocouples for measuring the gas temperature were chromel/alumel type with a $0.5 \mathrm{~mm}$ diameter stainless steel sheath. The leads of thermocouple hot junctions were supported by a $2 \mathrm{~mm}$ diameter brass tube offset by $10 \mathrm{~mm}$ from the axis of the cylinder. Assuming a Nusselt number of 2.0 and stainless steel properties for the sheathed junction, it was estimated that the time response for each of the thermocouples to measure gas temperature will be less that 0.6 seconds even if the hydrogen gas velocity is low. For experiments done with nitrogen gas the response time should be shorter than 4 seconds and for argon less than 6 seconds.

It is important to note that the heat flux and temperature of the copper strip in this experiment will be somewhat different to the expected heat flux and temperature to the 


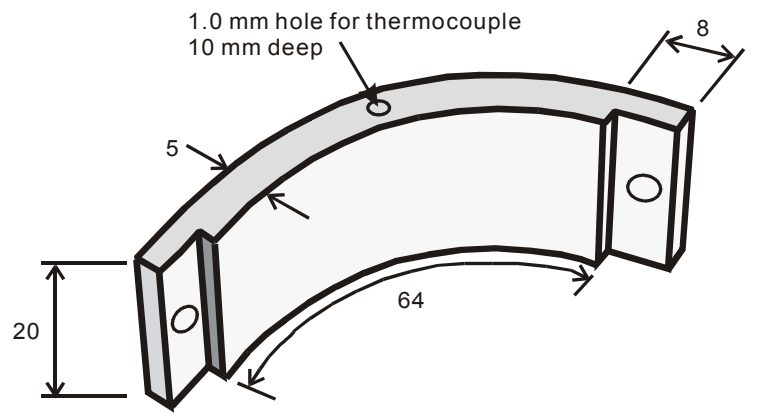

Fig. 2 Geometry of copper strip used for simultaneous measurement of heat flux and wall temperature (units: $\mathrm{mm}$ )

stainless steel wall if the PVC tube and copper strips were absent. However we do expect that the measured heat transfer coefficients may be similar to the case of the bare cylinder. This is because we expect the flow field will be similar and aligning the strips in a row should make the thermal boundary layer develop in a manner that is somewhat similar to the case of a bare cylinder. We verified this using the present result for the heat transfer coefficient to calculate and compare gas temperatures measured in an experiment with no PVC tube or strips inserted.

\subsection{Experimental procedure}

Gas and wall temperatures are recorded using a 16 channel amplifier connected to an analog/digital conversion card installed in a personal computer. Temperatures are logged at a rate of 50 per second. Hydrogen, nitrogen or argon is supplied from a cylinder regulated to maintain a $10 \mathrm{MPa}$ supply. The temperature time gradient of the copper strips used in determining the heat flux was based on least-squares quadratic fitting to two seconds of data. Experiments were repeated opening the valve different amounts to achieve different filling times. Discharging experiments were performed by recording temperatures and pressure during release of the gas to atmospheric pressure. Measurements were done using nitrogen gas, argon gas and hydrogen gas. As a preliminary measure, data where the calculated uncertainty was greater than $50 \%$ for any space position was automatically rejected from the analysis.

\subsection{Uncertainty analysis}

Uncertainty analysis followed the classical approach of Kline and McClintock ${ }^{(16)}$ as explained in detail by Moffat ${ }^{(17)}$. The heat transfer coefficient is defined by Eq. (1).

$$
h=\frac{q}{\left|T_{b}-T_{w}\right|}=\frac{\rho c(V / A)\left(d T_{w} / d t\right)}{\left|T_{b}-T_{w}\right|}
$$

In Eq. (1) $h$ is the heat transfer coefficient, $T_{\mathrm{b}}$ is the instantaneous bulk temperature of the gas, $T_{\mathrm{w}}$ is the temperature of the copper strip, $\rho$ is the density, $c$ is the specific heat, $V$ is the volume and $A$ the inside surface area of the copper strip.

The largest source of uncertainty is the estimate of the bulk temperature, $T_{\mathrm{b}}$, which was based on three readings as shown in Fig. 1. For the present analysis, the standard deviation for the three readings multiplied by the corresponding Student's $t$-value for $95 \%$ confidence was used to estimate the uncertainty. The next largest source of uncertainty was $\left(\mathrm{d} T_{\mathrm{w}} / \mathrm{d} t\right)$ used for determining the heat flux. Assuming each individual temperature reading has an uncertainty of 0.2 Kelvin, the method of perturbations ${ }^{(17)}$ was used to obtain the uncertainty in the temperature gradient. The effect of changing the number of points used to estimate 


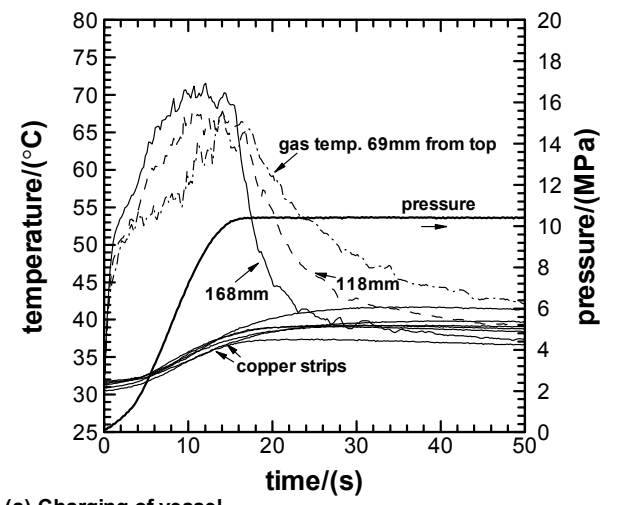

(a) Charging of vessel

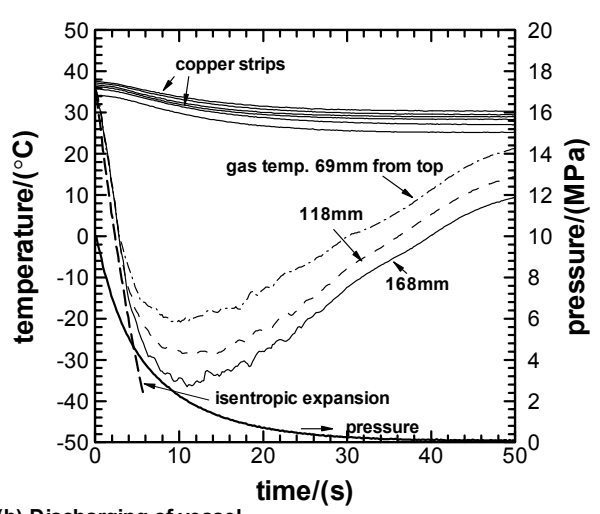

(b) Discharging of vessel

Fig. 3 Raw data from typical run of experiment using hydrogen

the slope was also included by recalculation with half the specified number. The uncertainty in the geometry in Fig. 2 was taken to be $\pm 0.2 \mathrm{~mm}$.

The Nusselt number, the Reynolds number and the Rayleigh number were defined as given in the nomenclature. All properties were evaluated at the bulk temperature and the uncertainties in estimating the Rayleigh and Reynolds numbers resulted mostly from the uncertainty in the pressure measurement which was experimentally determined to be about $\pm 30 \mathrm{kPa}$. Properties of hydrogen were based on data given in Refs. ${ }^{(18)-(20)}$. The uncertainty in the fundamental property data was not considered in the present analysis. The mass in the vessel at any instant in time was determined from the pressure measurement and bulk temperature. This supplied the necessary data to determine the rate of change of mass, $\dot{m}$, required for estimating $R e$. The uncertainty for the mass flow rate was calculated in a similar way to uncertainty for the temperature gradient in Eq. (1).

\section{Results}

\subsection{Measured temperatures}

Figure 3 shows typical data collected during the experiment. Figure 3(a) shows the case for charging of the vessel. As the pressure increases, the gas temperature rises from an initial temperature of around $30{ }^{\circ} \mathrm{C}$ to over $65{ }^{\circ} \mathrm{C}$. This is because the supplied gas does work compressing the gas in the vessel. For the first couple of seconds the gas temperature rises almost uniformly. Notice that during the first 15 seconds the gas temperature furthest from the inlet is the highest but after the pressure settles to a constant value of around 10 $\mathrm{MPa}$, the highest gas temperature is closest to the top of the vessel. The latter behavior is the result of buoyancy. The lower gas temperatures close to the inlet during the first 15 seconds may be attributed to the influence of the lower inlet gas temperature. Note that the inlet velocity gradually decreases with time and except for the first couple of seconds, the effect of the inlet gas temperature may hardly reach to the position furthest from the inlet. Notice also that the gas temperature drops quite quickly after the vessel reaches a steady pressure. This is the result of natural convection heat transfer to the wall since the flow into the vessel has almost stopped.

The temperatures of the copper strips are much lower than the gas temperatures in Fig. 3(a). This is desirable since a reasonably large temperature difference is required for the denominator in Eq. (1). Using the present technique the data in Fig. 3(a) beyond about 20 seconds does not give a useful estimate of the heat transfer coefficient.

Figure 3(b) shows typical data during a discharging experiment. The gas temperature 


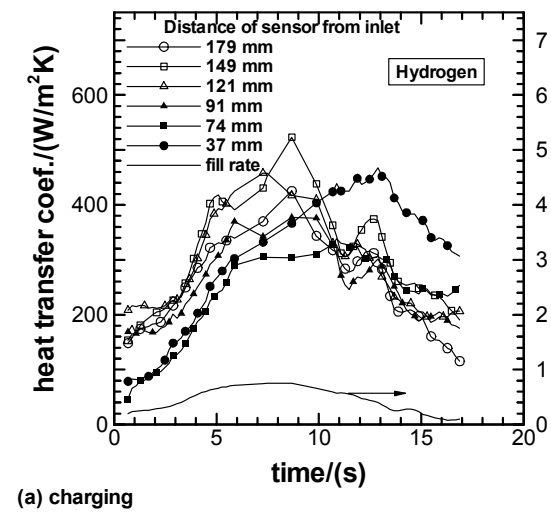

(a) charging

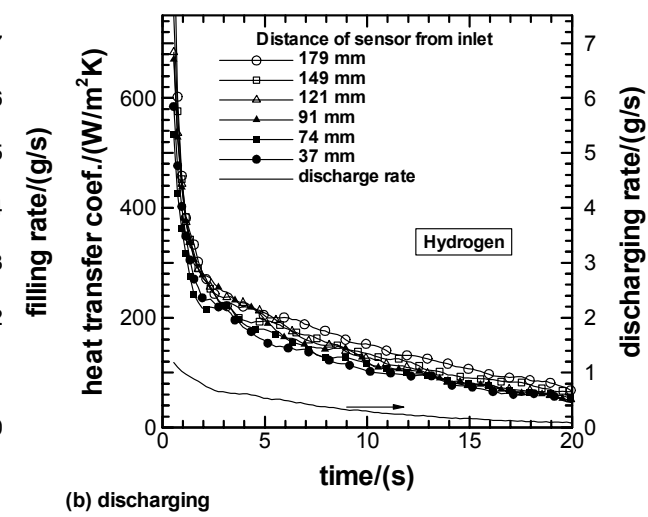

(b) discharging

Fig. 4 Measured local heat transfer coefficients for different axial positions

becomes quite low due to the work done pushing gas out of the vessel. For comparative purposes, a dashed line showing the temperature expected for isentropic expansion is also plotted in Fig. 3(b). During the first four seconds the measured gas temperatures approximately follow this line. This indicates that initially heat transfer from the wall is negligible in comparison with the cooling effect due to expansion at the sensor locations. However, as the pressure drop rate decreases, heat transfer starts to dominate and the gas temperature begins to rise by about 10 seconds. Due to buoyancy, the gas temperature distribution in the vessel becomes stratified as time progresses. The minimum temperature of the gas during the experiment is a strong function of the rate at which discharging takes place. The lowest gas temperature for all experiments was around $-100{ }^{\circ} \mathrm{C}$ for a case with hydrogen where the discharging time was about 3 seconds. Conversely, among all the experiments for charging of the vessel, the maximum gas temperature of around $85{ }^{\circ} \mathrm{C}$ occurred when the vessel was filled with hydrogen within about 5 seconds. The data for nitrogen and argon did not reach these extremes but the trends shown in Fig. 3 for hydrogen were almost the same for nitrogen and argon gas experiments. In general, the heat transfer coefficients for nitrogen and argon were significantly lower than for hydrogen under similar filling conditions. It may be worth mentioning finally that the compression process for gas charging is always irreversible (even without consideration of heat transferred to the wall) since the temperature of the gas entering is different to the temperature of the gas in the vessel. On the other hand, the expansion process during discharging may be considered isentropic for the gas remaining in the vessel when heat transfer between the wall and the gas is negligible.

\subsection{Measured heat transfer coefficients}

Figure 4 gives the heat transfer coefficients determined from the data in Fig. 3. For this particular case during charging, values range from less than $100 \mathrm{~W} / \mathrm{m}^{2} \mathrm{~K}$ to around 450 $\mathrm{W} / \mathrm{m}^{2} \mathrm{~K}$. It is also worth noting that the overall trend of heat transfer coefficient with time in Fig. 4(a) approximately follows the filling rate. This is also true for Fig. 4(b).

In Fig. 4(a) it is a little difficult to distinguish a clear spatial trend in the distribution of the heat transfer coefficient along the wall. During the first eight seconds, the heat transfer coefficient tends to be lower near the top of the vessel (dark symbols), however by about twelve seconds the trend starts to reverse and then finally by about 17 seconds the reversal is clearer. It seems reasonable to suppose that this changeover is related to the reversal of the gas temperature distribution shown in Fig. 3(a) at around 15 seconds.

For the discharging case shown in Fig. 4(b) there is a clear tendency for the heat transfer coefficient to be higher towards the bottom of the vessel (non-shaded symbols). This should be expected for natural convection from warm walls to a cold fluid since the 


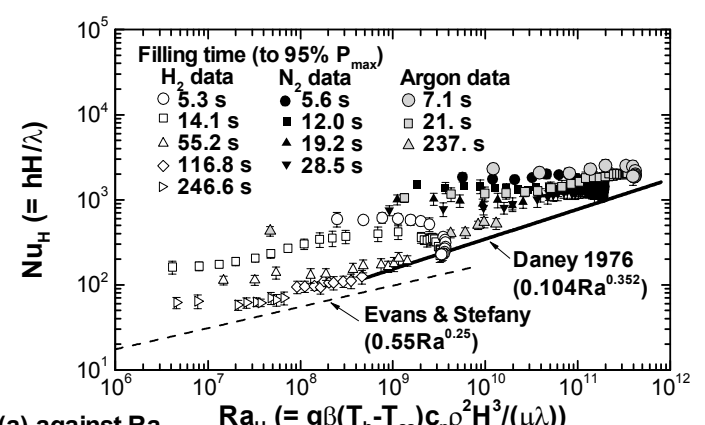

(a) against $\mathrm{Ra}$

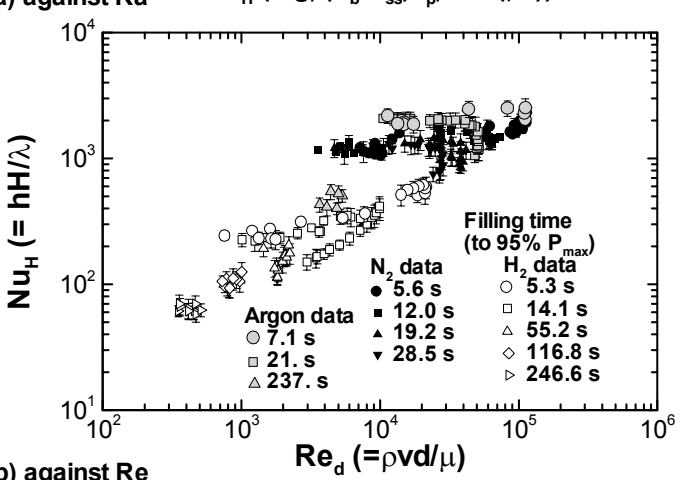

(b) against $\mathrm{Re}$

Fig. 5 Space-averaged Nusselt numbers during charging of the vessel

thermal boundary layer should begin growth from the bottom upwards. Nevertheless, on the whole the change in the space distribution for the heat transfer coefficient along the wall for the six positions is not more than about $30 \%$ of the mean value at any instant in time during discharging.

Another important feature of Fig. 4(b) is the high heat transfer coefficients during the first couple of seconds. A similar phenomenon has been observed by Evans and Stefany (7). In their study the wall temperature was changed suddenly and they attribute the high initial heat transfer coefficients to heat conduction into the fluid before convection effects dominate. Unlike their study, in the present experiment the fluid temperature changes more quickly than the wall. However, it is reasonable to suppose that the high initial heat transfer coefficients in Fig. 4(b) are also related to transient behavior as natural convection currents begin to form. For transient development of confined natural convection flows, Lin and Armfield ${ }^{(8)}$ make use of the following time scaling where $R a$ is the Rayleigh number based on the height and initial temperature difference and $a$ is the thermal diffusivity of the gas.

$$
\tau=\frac{t}{\left(H^{2} /\left(a R a^{1 / 2}\right)\right)}
$$

In their study they showed that convection currents become well developed when $\tau \approx$ $2 .{ }^{\left({ }^{(8)}\right.}$. For the case in Fig. 4(b) $\tau=2.0$ at about $t=1.3$ seconds. It is important to make note of this criterion since data collected in the present experiment for discharging of hydrogen could be correlated reasonably well, independent of time, provided the initial data with $\tau<2$ was omitted from the analysis.

\subsection{Correlation for heat transfer during charging of vessel}

As shown in Fig. 4(a) it was a little difficult to establish a clear trend for the space distribution of the Nusselt number. Therefore, rather than concentrate on the detailed distribution we decided to make a correlation for the average Nusselt number over the wall. This averaged Nusselt number is the main interest for the global model previously 


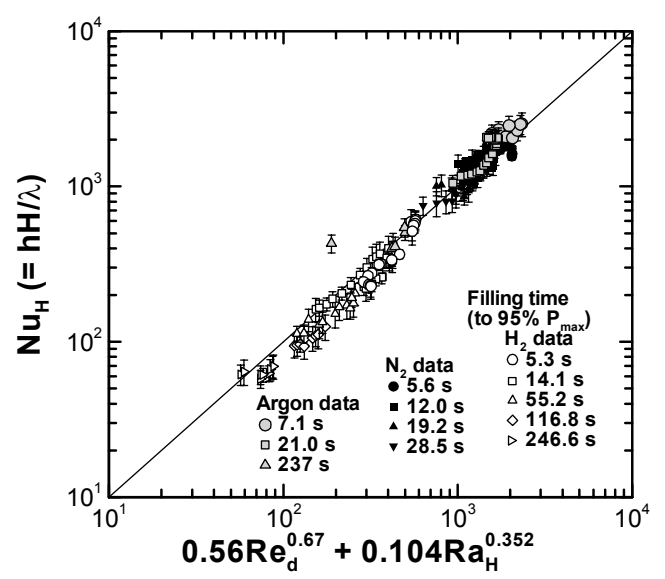

Fig. 6 Mixed convection correlation for charging vessel

developed ${ }^{(5)}$. For each point in time, the coefficient was taken to be the average value for the 6 measurement positions shown in Fig. 1. Figure 5(a) gives the data plotted against the Rayleigh number for experiments performed at different filling rates. Since the filling rate was not constant in any experiment the data is classified according to how much time was required to reach $95 \%$ of the maximum pressure. Figure 5(b) gives the same data plotted against the Reynolds number. Clearly the data cannot be described as a simple function of either $R a$ or $R e$. This indicates a mixed-convection situation.

For comparison with the present data, two correlations from the literature, developed for pure natural convection are also plotted in Fig.5(a). The correlation by Daney ${ }^{(6)}$ was developed from data measured using cryogenic liquids in containers of different shapes and orientations. It is appropriate for turbulent natural convection in enclosures. Notice that the Rayleigh number is to the power of 0.352 which is close to $1 / 3$. This makes the heat transfer coefficient almost independent of the characteristic length, $H$. Therefore it is meaningful to use Daney's relation in Fig. 5 even though he used $D$ as the characteristic length. The other correlation by Evans and Stefany ${ }^{(7)}$ gives the Nusselt number as a function of the $1 / 4$ power of the Rayleigh number. This is typical for laminar natural convection. Quite clearly from Fig. 5(a) these two correlations form a lower boundary for the present data. It is reasonable to suppose that the higher Nusselt numbers are obtained from shorter filling cases in the present experiment and are due to a contribution from forced convection. For the longer filling cases the data appears close to the Evans and Stefany Equation.

For empirical correlation of mixed convection heat transfer, one possible approach is to use an equation of the form given by Eq. (3) where $n$ is a positive number typically around three ${ }^{(21)}$.

$$
N u^{n}=N u_{\text {forced }}^{n} \pm N u_{\text {natural }}^{n}
$$

The natural convection contribution is added or subtracted depending on whether the buoyancy assists or opposes the forced convection heat transfer. Equation (3) has a desirable feature in that it also can be applied when the heat transfer is dominated either by forced or natural convection. Assuming that Daney's relation ${ }^{(6)}$ gives a suitable estimate for the natural convection contribution and that forced convection is correlated with $C R e^{b}$ then Eq. (4) gives a possible form for the present mixed convection situation. The effect of the Prandtl number, $P r$, on heat transfer is excluded here since the values of $\mathrm{Pr}$ for $\mathrm{H}_{2}$ and $\mathrm{N}_{2}$ are almost the same.

$$
N u^{n}=\left(C \operatorname{Re}^{b}\right)^{n}+\left(0.104 R a^{0.352}\right)^{n}
$$




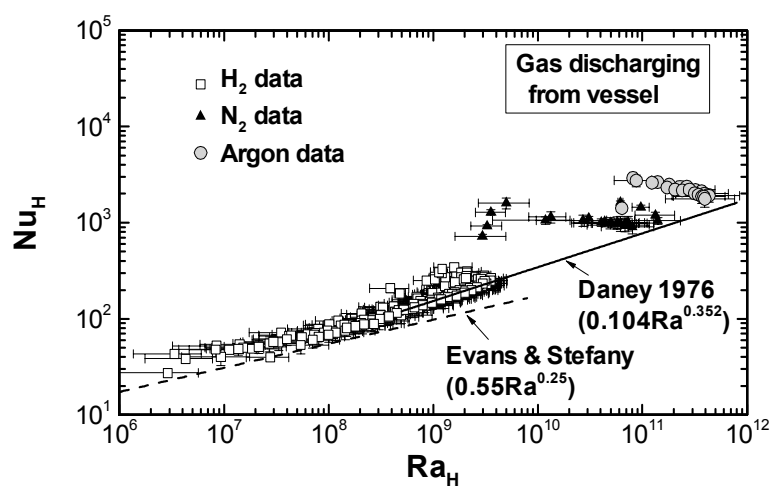

Fig. 7 Nusselt numbers during discharging as a function of Rayleigh number

The least-squares method was used to find values for $C$ and $b$ for the present data with different values of $n$. Equation (5) gives the recommended correlation for heat transfer during charging the cylinder. All properties are evaluated at the bulk fluid temperature.

$$
N u_{H}=0.56 \mathrm{Re}_{d}^{0.67}+0.104 R a_{H}^{0.352}
$$

As indicated above, Eq. (5) was developed from the present data for a vertical cylinder with the inlet at the top, aspect ratio: $H / D=2.8$ and $D / d=7.5$ (see Fig. 1). It is applicable over the range of the Reynolds and Rayleigh numbers given in Eqs. (6a) and (6b).

$$
\begin{gathered}
3 \times 10^{5}<R a_{H}<4.2 \times 10^{11} \\
270<\operatorname{Re}_{d}<1.2 \times 10^{5}
\end{gathered}
$$

Comparing Eqs. (4) and (5) it is clear that we have selected $n=1$. Using higher values of $n$ did not lead to a better correlation with the data so we decided to keep the simplest choice. Also using coefficients other than those proposed by Daney for the natural convection term did not result in a much better correlation.

Figure 6 shows how well Eq. (5) approximates the data from the present experiment. For most of the data points the equation is within range of the uncertainty. Comparing Fig. 6 with Fig. 5 it is clear that mixed convection is the most appropriate treatment of the data.

\subsection{Correlation for heat transfer during discharging of vessel}

Figure 7 shows data collected for the Nusselt number during discharging, plotted against the Rayleigh number. Note that all data for dimensionless time, $\tau<2.0$ has been omitted from the analysis. Apart from some data for nitrogen, the bulk of the data can be approximated reasonably well with Daney's relation [6], which is given in Eq. (7). This suggests that the contribution to the flow field resulting from the gas leaving the vessel is not large and heat transfer is dominated by natural convection. It should be expected that the natural convection contribution will be similar in both heating and cooling experiments ${ }^{(6),(7)}$. Also during charging, the jet of gas entering is more likely to create turbulence and enhance the heat transfer than for the case of discharging flow.

Unlike the charging case, the data for discharging could not be improved by a mixed convection-type correlation. Therefore we recommend Eq. (7) for calculating the heat transfer coefficient during discharging.

$$
N u_{H}=0.104 R a_{H}^{0.352}
$$

From the present experiment most of the data not captured by Eq. (7) are for times less than about three seconds from when discharging commenced when the gas temperature and pressure were changing rapidly. As already mentioned in section 3.2 the high heat transfer coefficients near the beginning may be attributed to transient behavior corresponding to the 
start-up of the convection currents. For this reason we have not included the data where $\tau$ $<2.0$ in Fig. 7. This may be sufficient for most of the hydrogen data, but it cannot account for all of the nitrogen and argon data since some data far from the line remain in Fig. 7. For nitrogen and argon, the response time of the thermocouples measuring the gas temperature may be the problem. As mentioned in section 2.1 the response time of the sensors could be as large as 4 seconds for nitrogen and 6 seconds for argon if the gas velocity is low. This effect was not included in the error analysis. Since the gas temperature drops rapidly (see Fig. 3(b)), the Nusselt number may be over-estimated and the Rayleigh number under-estimated. This 'response time' problem may not be as severe in the charging case if we suppose that the sensor is in a high-velocity gas stream caused by the jet of gas entering the vessel. In any case, based on the present study we tentatively recommend Eq. (7) for discharging with the qualification that $\tau>2.0$ where $\tau$ is defined in Eq. (2).

\section{Conclusions}

The main conclusions may be summarized as follows.

1. During charging the heat transfer in the pressure vessel is best described as mixed convection.

2. A correlation was developed for mixed convection during charging of the cylinder for the three different gases independent of time (Eq. (5)).

3. Natural convection dominates the heat transfer during discharging and most data for hydrogen could be correlated reasonably well with Eq. (7).

4. Much of the data for nitrogen and argon during discharging suggested higher heat transfer rates than indicated by Eq. (7). Although this may be partly due to convective enhancement, we could not rule out the possibility of an experimental error due to the slower response time of the sensors in the gases with lower thermal conductivity.

5. During the first one or two seconds the measured heat transfer coefficient was consistently higher than that for the remainder of the data. This can be attributed partly to the transient start-up of the convection currents.

6. The local heat transfer coefficient was consistently higher towards the bottom of the vessel during discharging. During charging the trend was not so clear. Initially the heat transfer coefficient was higher near the bottom but as the filling progressed the trend reversed.

\section{References}

(1) S. M. Aceves, J. Martinez-Frias, O. Garcia-Villazana, Analytical and experimental evaluation of insulated pressure vessels for cryogenic hydrogen storage. International Journal of Hydrogen Energy, Vol. 25 (2000) pp. 1075-1085.

(2) L. Schlapbach, A. Zuttel, Hydrogen-storage materials for mobile applications, Nature, Vol. 414 (2001) pp. 353-358.

(3) A. Zuttel, Materials for hydrogen storage, Materials Today (2003) pp. 24-33.

(4) F. C. Shen, A filament-wound structure technology overview, Materials Chemistry and Physics, Vol. 42 (1995) pp. 96-100.

(5) M. Monde, Y. Mitsutake, P. Woodfield, S. Maruyama, Characteristics of heat transfer and temperature rise of hydrogen during rapid hydrogen filling at high pressure, Heat Transfer - Asian Research, Vol. 36 (2007) pp. 13-27.

(6) D. E. Daney, Turbulent natural convection of liquid deuterium, hydrogen and nitrogen within enclosed vessels, International Journal of Heat and Mass Transfer, Vol. 19 (1976) pp. 431-441.

(7) L. B. Evans, N. E. Stefany, An experimental study of transient heat transfer to liquids in 
cylindrical enclosures, Chemical Engineering Progress Symposium Series, American Institute of Chemical Engineer, Vol. 62, No. 64 (1966) pp. 209-215.

(8) W. Lin, S. W. Armfield, Natural convection cooling of rectangular and cylindrical containers, International Journal of Heat and Fluid Flow, Vol. 22 (2001) pp. 72-81.

(9) W. Lin, S. W. Armfield, Direct simulation of natural convection cooling in a vertical circular cylinder, International Journal of Heat and Mass Transfer, Vol. 42 (1999) pp. 4117-4130.

(10) A. Lemebre, J. P. Petit, Laminar natural convection in a laterally heated and upper cooled vertical cylindrical enclosure, International Journal of Heat and Mass Transfer, Vol. 41 (1998) pp. 2437-2454.

(11) H. S. Kwak, K. Kuwahara, J. M. Hyun, Convective cool-down of a contained fluid through its maximum density temperature, International Journal of Heat and Mass Transfer, Vol. 41 (1998) pp. 323-333.

(12) M. Holzbecher, A. Steiff, Laminar and turbulent free convection in vertical cylinders with internal heat generation, International Journal of Heat and Mass Transfer, Vol. 38 (1995) pp. 2893-2903.

(13) E. Papanicolaou, V. Belessiotis, Transient natural convection in a cylindrical enclosure at high Rayleigh numbers, International Journal of Heat and Mass Transfer, Vol. 45 (2002) pp. 1425-1444.

(14) J. D. Jackson, M. A. Cotton, B. P. Axcell, Studies of mixed convection in vertical tubes, International Journal of Heat and Fluid Flow, Vol. 10 (1989) pp. 2-15.

(15) J. D. Jackson, Studies of buoyancy influenced turbulent flow and heat transfer in vertical passages, KN-24, Proceedings of 13th International Heat Transfer Conference, Sydney, August 2006.

(16) S. J. Kline, F. A. McClintock, Describing uncertainties in single-sample experiments, Mechanical Engineering (1953) pp. 3-8.

(17) R. J. Moffat, Describing the uncertainties in experimental results, Experimental Thermal and Fluid Science (1988) pp. 3-17.

(18) R. D. McCarty, J. Hord, H. M. Roder, Selected Properties of Hydrogen (Engineering Design Data) (monograph), U.S. National Bureau of Standards (1981).

(19) R. H. Perry, D. W. Green, Perry's Chemical Engineers' Handbook, 7th Edition, McGraw Hill, New York, 1997.

(20) M. J. Assael, S. Mixafendi, W. A. Wakeham, The viscosity and thermal conductivity of normal hydrogen in the limit of zero density, Journal of Physical and Chemical Reference Data, Vol. 15 (1986) pp. 1315-1322.

(21) F. P. Incropera, D. P. DeWitt, Fundamentals of Heat and Mass Transfer, 3rd Edition, Wiley, Singapore, 1990 pp. 566-567. 